\title{
Connectivity Reliability on an Urban Rail Transit Network from the Perspective of Passenger Travel
}

\author{
Jie Liu ${ }^{1} \cdot$ Qiyuan Peng ${ }^{1} \cdot J_{i n q u}$ Chen $^{1} \cdot$ Yong Yin $^{1}$
}

Received: 20 June 2019/Revised: 22 October 2019/Accepted: 30 October 2019/Published online: 9 December 2019

(C) The Author(s) 2019

\begin{abstract}
Under the background of urbanization and the rapid development of urban rail transit (URT), serious attention has been focused on URT network reliability in recent years. In this work, in order to measure network reliability, three indicators are constructed based on passengers' tolerable travel paths, passenger travel efficiency and passenger travel realization on a URT network. The passenger tolerability coefficient, which is the ratio of passengers' tolerable travel time to the shortest possible travel time, is proposed and added to the indicators. It reflects passengers' behavior with respect to choice of travel paths. The ratio of affected passenger volume (RAPV) is proposed to identify important stations. Finally, the connectivity reliability of Wuhan's subway network is analyzed by simulating attacks on stations. The results show that the degree centrality, betweenness centrality and RAPV indicators of stations can effectively identify the important stations that have a significant impact on the connectivity reliability of the network. In particular, the RAPV
\end{abstract}

Yong Yin

yinyong@home.swjtu.edu.cn

Jie Liu

JieLiu.SWJTU@outlook.com

Qiyuan Peng

qiyuan-peng@swjtu.edu.cn

Jinqu Chen

Chenjinqu@my.swjtu.edu.cn

1 National United Engineering Laboratory of Integrated and Intelligent Transportation, National Engineering Laboratory of Integrated Transportation Big Data Application Technology, School of Transportation and Logistics, Southwest Jiaotong University, Chengdu 610031, Sichuan, China

Communicated by Xuesong Zhou. indicator effectively identifies stations that have the greatest influence on passenger travel realization. The connectivity reliability of Wuhan's subway network is sensitive to passenger tolerability coefficient, and reliability is greater during non-peak hours than during peak hours. In addition, the stations that are important to the connectivity reliability of the Wuhan subway have two features, i.e., they are located at the center of the city, and they are important for connecting subgraphs of the network.

Keywords Urban rail transit network - Connectivity reliability $\cdot$ Passenger travel - Tolerable travel paths . Travel efficiency $\cdot$ Travel realization

\section{Introduction}

In recent years, China has experienced rapid urbanization. Urban rail transit (URT), with the advantages of large capacity, low energy consumption and environmental protection, has grown rapidly. Now, China has the longest URT operating lines in the world. However, certain emergency events (such as natural disasters or operational accidents) can cause great harm to the normal operations of a URT line. Passenger travel time increases and travel paths are disrupted because of these emergencies. In addition, a URT network is a small-world network [1-3], in which the number of links between stations is small, and thus the connectivity is easily affected by emergencies. Therefore, efficient and comprehensive methods for evaluating the connectivity reliability of a URT network and identifying important stations with a large impact on connectivity are of great significance for improving network reliability and ensuring normal operations.

Researchers have proposed various effectiveness measures for analyzing transportation reliability. Connectivity 
reliability [4], travel time reliability [5] and capacity reliability [6] have been widely used to measure the reliability of transportation networks. Connectivity reliability is a measure of the number of connected nodes in a transportation network. Passenger travel between nodes at any given time can occur only through nodes that are in a connected state. Therefore, connectivity reliability is the basis of travel time and capacity reliability.

Connectivity reliability was first proposed by Mine and Kaiwai [7]. Many researchers have studied the connectivity reliability of transportation networks based on graph theory and network topology. For example, Bell and Iida [8] and Wakabayashi and Iida [9] defined connectivity reliability as the probability that a connection between a pair of nodes exists when one or more links are removed. Jiang et al. [10] and Liu \& Song [11] studied the reliability of the Guangzhou subway network using complex network theory. The effects of removal of stations on network structure were analyzed by simulating random or deliberate attacks on stations. The authors found that removing stations randomly had little impact on the Guangzhou subway network structure. Wang and Xu [12] and Zhang et al. [13] adopted a similar method to analyze the reliability of a URT network and a high-speed railway network, respectively. Zhang et al. [14] studied the resilience of 17 principal networks in terms of connectivity. All of the above-mentioned studies primarily analyzed the connectivity reliability of networks based on network topology, the effects of removing nodes and links, without considering passenger effects. Other researchers have studied transportation network reliability while considering passengers, such as the work by Mattsson and Jenelius [15], who summarized and reviewed the existing research on transportation reliability. They pointed out that it is necessary to consider traffic demand and transport supply to determine reliability. Zhang et al. [16] proposed a level-of-service (LOS)-based evaluation model to calculate the connectivity reliability of a regional transportation network considering passenger volume. Liu et al. [17] analyzed the connectivity reliability of rail transit through Monte Carlo simulation. Guidotti et al. [18] proposed two types of indicators to measure the connectivity reliability of a transport network. The connectivity reliability of a URT network was compared through the use of indicators that considered weights of nodes and indicators that did not. Li et al. [19] proposed four connectivity reliability indicators, which were then weighted to establish a single indicator that was used to analyze the connectivity reliability of the Beijing subway network. Reggiani et al. [20] performed a more in-depth analysis on how resilience and vulnerability could be framed, interpreted and measured, and their relationship with connectivity.
Some researchers have used passenger trips between origin and destination (OD) pairs to determine the weights of station pairs. As a result, however, the effects of passengers' travel behaviors on transportation networks are not considered when analyzing the connectivity reliability of the transportation networks. In addition, many researchers assume that two nodes are connected as long as at least one connection path exists between the two nodes. However, this assumption is not entirely consistent with passenger behavior regarding choice of travel path. Passengers tend to choose the travel path whose travel time is shortest. If the shortest travel path corresponding to the shortest travel time cannot be used, passengers will choose other paths whose travel times are less than their tolerable travel times. If the travel times of all connected paths are longer than their tolerable travel times, even though the paths are connected, passengers will not use them.

In the present work, indicators are proposed for measuring the connectivity reliability of a URT network and identifying important stations on the network by drawing on the above-mentioned literature. The main research contributions can be summarized as follows:

- Three indicators are proposed to reflect passengers' travel behavior: passengers' tolerable travel paths, passenger travel efficiency and passenger travel realization on a URT network.

- The ratio of affected passenger volume (RAPV) is proposed to measure the importance of stations on the URT network.

- The important stations are identified based on their degree centrality (DC), betweenness centrality (BC), closeness centrality (CC) and ratio of affected passenger volume (RAPV). The characteristics of important stations are analyzed based on passenger volume, location of stations and morphological characteristics of the URT network.

- The connectivity reliability of Wuhan's subway network is analyzed via simulation of attacks on stations. The sensitivity of the passenger tolerability coefficient to the three indicators of connectivity reliability of the Wuhan subway is analyzed.

\section{Method}

To study the connectivity reliability of a URT network, the network is defined as a directed graph. Passengers tend to choose the travel paths whose travel times are tolerable. Those travel paths are defined as tolerable travel paths. Three indicators are put forward to measure the connectivity reliability of a URT network based on passengers' tolerable travel paths, passenger travel efficiency and 
passenger travel realization on the network. To identify the important stations, the RAPV is proposed. A user stochastic equilibrium model with link capacity constraints is used to calculate the RAPV.

\subsection{Network Definition}

The URT network can be defined as a directed graph $G(N, E)$, where $N$ represents the set of stations, and $E \subseteq$ $\{(x, y) \mid x, y \in N, x \neq y\}$ represents the set of links connecting stations directly. There are multiple connected paths from one station to another. It is assumed that path ${ }_{\mathrm{od}}$ is the set of connected path(s) from station $o$ to station $d$. The connected path $i$ from station $o$ to station $d$ includes the sets of transfer station(s) $M_{o d}^{i}$, non-transfer station(s) $N_{o d}^{i}$ and links $E_{o d}^{i}$.

\subsection{Passengers' Tolerable Travel Paths}

As mentioned above, not all the connected paths are meaningful for passengers. Passengers will choose to use such a path only when it is tolerable. To calculate the number of tolerable travel paths from one station to another, the passenger tolerability coefficient $\alpha$ is put forward. Equation (1) is used to confirm whether path $i$ from station $o$ to station $d$ is a tolerable travel path:

$n\left(t_{o d}^{i}, t_{o d}^{\text {shortest }}\right)= \begin{cases}1 ; & t_{o d}^{i} / t_{o d}^{\text {shortest }} \leq \alpha \\ 0 ; & \text { otherwise }\end{cases}$

Here, $n\left(t_{o d}^{i}, t_{o d}^{\text {shortest }}\right)$ is a $0-1$ variable, and $t_{o d}^{i}$ is the travel time of path $i$ from station $o$ to station $d$. $t_{o d}^{\text {shortest }}=$ $\min \left\{t_{o d}^{i} \mid i \in\right.$ path $\left._{o d}\right\}$ is the shortest travel time from station $o$ to station $d$. If path $i$ is a tolerable travel path, then $n\left(t_{o d}^{i}, t_{o d}^{\text {shortest }}\right)=1$; otherwise $n\left(t_{o d}^{i}, t_{o d}^{\text {shortest }}\right)=0 . \alpha$ is the passenger tolerability coefficient. It reflects the relationship between tolerable travel time and the shortest possible travel time.

$t_{o d}^{i}$ can be calculated by Eq. (2). It includes the train running time on links (including the train start and stop time), train dwell time, passenger transfer time and passenger waiting time.

$t_{\text {od }}^{i}=\sum_{e \in E_{o d}^{i}} t_{e}+\sum_{m \in M_{o d}^{i}} t_{m}^{\mathrm{dwell}}+\sum_{n \in N_{o d}^{i}} t_{n}+t_{\text {wait }}^{o}$,

where $e$ and $E_{o d}^{i}$ are link $e$ and the set of link(s) on path $i$ from station $o$ to station $d$, respectively. $t_{e}$ is the train running time on link e. $t_{m}^{\mathrm{dwell}}$ is the train dwell time at nontransfer station $m$, where $m \in M_{o d}^{i}$. $t_{n}$ is the transfer time at transfer station $n$, where $n \in N_{o d}^{i}$. $t_{\text {wait }}^{o}$ is the waiting time at the station of origin which can be estimated as half of the headway.

\subsection{Evaluating the Connectivity Reliability of a URT Network}

To measure the connectivity reliability of a network, the network efficiency (the mean of the reciprocal of the shortest distance between all nodes), the maximum connected subgraph and other derivative indicators are used by researchers [10-14, 17]. These indicators lack information on passenger volume and the number of tolerable travel paths. The passenger volume of each station in a URT network varies greatly. Therefore, the transportation role and the weights of stations differ. The ratio of passenger volume entering and exiting a station to the passenger volume of a URT network is used as the weight of the station. Three indicators that consider the weights of stations and the number of tolerable travel paths between pairs of stations are proposed to evaluate the connectivity reliability of a URT network.

\subsubsection{Number of Tolerable Travel Paths on a URT Network}

Some tolerable travel paths on a URT network become unavailable when operational accidents, terrorist attacks or natural disasters occur. In this case, the connectivity reliability of the network decreases. Therefore, the higher the number of tolerable travel paths between pairs of stations, the higher the probability that passengers can travel between pairs of stations. The average number of tolerable travel paths for every passenger is used to evaluate the connectivity reliability of a URT network before the network is destroyed. It is represented as Eq. (3):

$R_{\text {path }}^{0}=\frac{\sum_{o \in N} \sum_{d \in N, o \neq d} \sum_{i \in \text { path }_{o d}} v_{o d} \cdot n\left(t_{o d}^{i}, t_{o d}^{\text {shortest }}\right)}{V}$

where $R_{\text {path }}^{0}$ is the average number of tolerable travel paths for every passenger before the network is damaged, $N$ is the set of stations in the URT network, $v_{o d}$ is the passenger volume from station $o$ to station $d$, and $V$ is the passenger volume in the network.

After a URT network is damaged, the average number of tolerable travel paths for every passenger will decrease. It is assumed that the network suffered a damage event $\delta$, causing $x$ number of stations to lose their functioning. In this situation, the average number of tolerable travel paths for every passenger $R_{\text {path }}^{(\delta, x)}$ is calculated by Eq. (3). The relative number of tolerable travel paths $R_{\text {path }}$ is used to measure the connectivity reliability of the network after it is destroyed. It can be calculated by Eq. (4):

$R_{\text {path }}=\frac{R_{\text {path }}^{(\delta, x)}}{R_{\text {path }}^{0}}$. 


\subsubsection{Travel Efficiency of a URT Network}

The connectivity reliability of a URT network is always measured based on network efficiency. The implicit assumption of network efficiency is that every station plays the same role in the network (the weights of stations are the same). However, the functions of stations in a URT network are different, and the passenger volume between pairs of stations varies considerably. Therefore, passenger volume between stations should be considered in calculating travel efficiency. In addition, network efficiency mainly reflects the connectivity reliability of the network from the shortest distance between nodes. URT passengers focus more on travel time. Therefore, the passenger volume and passenger travel time should be combined to measure the travel efficiency of a URT network. $E_{\text {eff }}^{0}$ is the travel efficiency of a URT network before the URT network is damaged, which is calculated by Eq. (5):

$E_{\mathrm{eff}}^{0}=\frac{1}{V} \sum_{o \in N} \sum_{d \in N, o \neq d} v_{o d} \cdot \frac{1}{t_{o d}^{\text {shortest }}}$.

After a URT network is damaged, the travel efficiency of the URT network decreases. The travel efficiency decreases to $E_{\mathrm{eff}}^{(\delta, x)}$ when the network has suffered damage $(\delta, x)$. The relative travel efficiency of a URT network $R_{\text {eff }}$ is used to measure the connectivity reliability of the network, which is calculated by Eq. (6):

$R_{\mathrm{eff}}=\frac{E_{\mathrm{eff}}^{(\delta, x)}}{E_{\mathrm{eff}}^{0}}$.

\subsubsection{Rate of Passenger Travel Realization on a URT Network}

Most passengers can travel on a URT network within tolerable travel time in normal operations. Thus the rate of passenger travel realization on the network is near $100 \%$. However, some passengers' tolerable travel paths are interrupted when a URT network is destroyed. Passengers choose alternative transportation, and the travel realization rate decreases. Therefore, the passenger travel realization rate on a URT network is put forward to measure the connectivity reliability of the network. This rate is represented as Eq. (7) after the network has suffered damage $(\delta, x)$.

$R_{\text {rate }}=\frac{V^{(\delta, x)}}{V}=\frac{\sum_{o \in N} \sum_{d \in N, o \neq d} v_{o d} \cdot n_{o d}^{(\delta, x)}}{V}$

where $R_{\text {rate }}$ is the rate of passenger travel realization on a URT network after the network has suffered damage $(\delta, x)$, $V^{(\delta, x)}$ is the number of passengers who travel on the network within tolerable travel time when the network has suffered damage, and $n_{o d}^{(\delta, x)}$ is a $0-1$ variable which is equal to 1 if there is at least one tolerable travel path from station $o$ to station $d$, and 0 otherwise.

\subsubsection{Identifying Important Stations}

A number of studies have been conducted to identify important nodes in complex networks and to identify important stations in URT networks [21-23]. Various indicators have been widely used to evaluate the importance of stations, including degree centrality (DC), betweenness centrality (BC) and closeness centrality (CC). DC emphasizes the number of direct links to the station. A station's BC is defined as the ratio of the number of shortest paths via the station to the number of shortest paths on the network. CC refers to distances between the evaluation station and other stations. However, these three indicators focus on identifying important stations based on the network topology. The impact of stations on passenger travel is neglected. For this purpose, the ratio of affected passenger volume (RAPV) is used to measure a station's importance. Supposing that some passengers' travel is affected by station $j$, the RAPV indicator for station $j$ is calculated by Eq. (8):

$I_{j}=\frac{\text { flow }_{j}}{V}$,

where $I_{j}$ is the RAPV value of station $j$, and flow is the $_{j}$ passenger flow that will be affected by station $j$.

In Eq. (8), to calculate the importance indicator of station $j$, the passenger flow via station $j$ needs to be confirmed. The traffic assignment model should be applied to determine the passenger flow via stations. Some studies allocate passenger trips on a URT network considering the constrained capacity caused by the train, platform and transfer corridor [24, 25], while others employ a user stochastic equilibrium model to allocate passenger trips on the network [26, 27]. In this paper, the RAPV of stations can be calculated simply by determining the passenger flow via stations. Considering that the user stochastic equilibrium model with the link capacity constraints is able to obtain the result quickly and with high accuracy, it is used to calculate the RAPV indicator, which is specified in Eqs. (9)-(15). The passenger flow via station $j$ can be calculated by Eq. (14), which corresponds to Eq. (8).

$$
\begin{aligned}
\min Z(f)= & \frac{1}{\theta} \sum_{o} \sum_{d} \sum_{k} v_{o d}^{k} \cdot \operatorname{Inv}_{o d}^{k} \\
& +\sum_{e} \int_{0}^{\text {flow }_{e}} \operatorname{flow}_{e}(\omega) \mathrm{d} \omega
\end{aligned}
$$

Subject to 
$\sum_{k} v_{o d}^{k}=v_{o d} \quad \forall o \in N, d \in N$

$v_{o d}^{k} \geq 0 \quad \forall o \in N, d \in N, k$

flow $_{e} \leq \operatorname{cap}_{e} \quad \forall e \in E$

flow $_{e}=\sum_{o} \sum_{d} \sum_{k} v_{o d}^{k} \cdot \delta_{e, k}^{o d} \quad \forall e \in E$

flow $_{j}=\sum_{o} \sum_{d} \sum_{k} v_{o d}^{k} \cdot \delta_{j, k}^{o d} \quad \forall j \in N$

flow $_{e}(\omega)=\left\{\begin{array}{ll}1.3 \cdot t_{e} ; & \omega \leq \text { seat }_{e} \\ \left(\frac{1.3 \cdot \text { seat }_{e}+2.32 \cdot\left(\omega-\text { seat }_{e}\right)}{\omega}\right) \cdot t_{e} ; & \omega>\text { seat }_{e}\end{array} \quad e \in E\right.$

where $\theta$ is a non-negative parameter which represents the accuracy of users' perceptions of cost, $v_{o d}^{k}$ is the volume of passengers who travel from station $o$ to station $d$ on path $k$, flow $_{e}$ is the passenger flow of link $e$. It can be calculated by Eq. (13). The link capacity constraint is shown in Eq. (12).

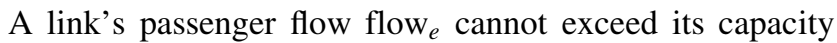
$\operatorname{cap}_{e}$. flow $(\omega)$ is the functional relation between the generalized cost and passenger flow $\omega$ for link $e$, which is expressed as Eq. (15). It specifies that crowded travel cost is related to whether passengers can sit or have to stand on the link. In terms of passengers' subjective perceptions, passengers perceive that the crowded travel cost of links is higher when they are standing than when they are sitting. Wardman and Whelan [28] proposed that if passengers can sit, then the crowded travel cost in a link is equal to 1.3 times the in-vehicle time of the link. Otherwise, it is equal to 2.32 times the in-vehicle time. $\delta_{e, k}^{o d}$ is a $0-1$ variable. If the path $k$ from station $o$ to station $d$ contains link $e$, then $\delta_{e, k}^{o d}=1$; otherwise $\delta_{e, k}^{o d}=0 . \delta_{j, k}^{o d}$ is a $0-1$ variable. If the path $k$ from station $o$ to station $d$ contains station $j$, then $\delta_{j, k}^{o d}=1$; otherwise $\delta_{j, k}^{o d}=0$.

\section{Implementation}

\subsection{Data Preparation}

The passenger travel data and train running data of Wuhan's subway network are used to verify the feasibility and effectiveness of the method and the indicators used. Figure 1 shows the operating lines, station names (shortened form) and numbers for the Wuhan subway in September 2018.

The automatic fare collection (AFC) data for 5 working days in September 2018 were obtained from the Wuhan subway operator, Wuhan Metro Co., Ltd. The data include the types of tickets, number of tickets, entry and exit time at stations, and station names and numbers. The number of tickets is matched to obtain the passenger volume from one station to another. The average passenger volume between pairs of stations is calculated to construct origin and destination (OD) matrices during peak and non-peak hours.

To calculate the travel time from one station to another, the travel time (including train dwell time and train running time) of links, headway for different lines and the transfer time at transfer stations are obtained from Wuhan Metro Co., Ltd. Tables 1 and 2 show the headway for lines and the transfer time at transfer stations during peak and nonpeak hours, respectively. The travel time for a link is the same during peak and non-peak hours. Table 3 shows the travel time for various links on line 1 . The travel time for other links is shown in Table 5 in the "Appendix".

\subsection{Important Stations}

The important station identification indicators (DC, BC, CC and RAPV) are calculated. The 10 most important stations in Wuhan's subway network are listed in Table 4.

Table 4 shows that four identification indicators have identified some of the same important stations, such as stations 21, 20, 23 and 48. However, the importance ranking of the four stations is different for the indicators. These four stations are transfer stations in Wuhan's subway network. To analyze the connectivity reliability of Wuhan's subway network and demonstrate the accuracy of different identification indicators, the influence of failure of important stations on connectivity reliability of the network is analyzed.

\subsection{Connectivity Reliability of Wuhan's subway network}

Serious accidents (such as natural disasters, terrorist attacks or operational accidents) often cause stations to close. The affected stations experience failure in this situation. To analyze the connectivity reliability of Wuhan's subway network, MATLAB is used to simulate attacks on stations to cause station failure. If a station is attacked, then the station and the links that are directly connected to the station are removed from the network. To analyze the impact of failure of important stations on the connectivity reliability of Wuhan's subway network, five attack modes are used to simulate station failures. The deliberate attack mode is used to attack important stations as determined by the importance ranking for stations identified by BC, DC, $\mathrm{CC}$ and RAPV indicators. The random attack mode is used to attack stations randomly without considering their importance. The values of connectivity reliability evaluation indicators [from Eqs. (3) to (7)] are calculated when 
Fig. 1 Wuhan's subway network in September 2018

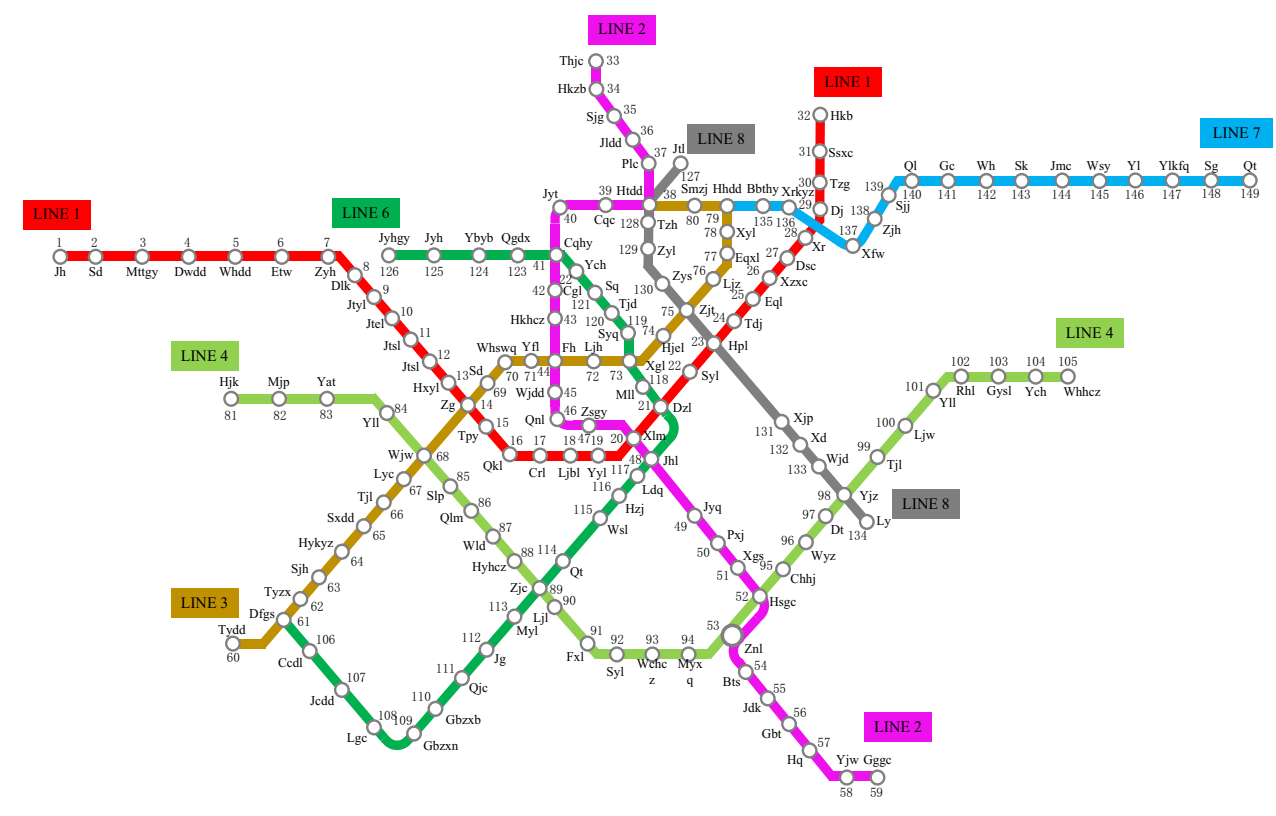

Table 1 Headway for lines during peak and non-peak hours

\begin{tabular}{llllll}
\hline \multirow{2}{*}{ Lines } & \multicolumn{2}{l}{ Downstream headway $(\mathrm{s})$} & & \multicolumn{2}{c}{ Upstream headway $(\mathrm{s})$} \\
\cline { 2 - 3 } \cline { 5 - 6 } \cline { 5 - 6 } & Peak hours & Non-peak hours & & Peak hours & Non-peak hours \\
\hline 2 & 225 & 390 & 277 & 420 \\
3 & 225 & 390 & 200 & 300 \\
4 & 257 & 450 & 225 & 390 \\
5 & 225 & 300 & 390 & 225 & 390 \\
6 & 257 & 420 & 200 & 300 \\
7 & 360 & 600 & 257 & 420 \\
\hline
\end{tabular}

every station is attacked in the simulation process. To determine the tolerable travel paths between the pairs of stations, the passenger tolerability coefficient must be confirmed. We conducted an in-depth questionnaire survey on the characteristics of passengers' travel path choices in Wuhan's subway network, which revealed the passenger tolerability coefficient $\alpha$ of 1.38 .

\subsubsection{Relative Number of Tolerable Travel Paths in Wuhan's subway network}

The connectivity reliability of Wuhan's subway network can be determined by the relative number of tolerable travel paths $R_{\text {path }}$. Attacking stations randomly and attacking important stations deliberately are used to simulate station failures during peak and non-peak hours. Before the stations are destroyed, the average number of tolerable travel paths for every passenger in the network is
3.49 and 4.16 during peak and non-peak hours, respectively. Figure $2 \mathrm{a}, \mathrm{b}$ shows simulation output for the relative number of tolerable travel paths $R_{\text {path }}$ under five attack modes during peak and non-peak hours, respectively. It can be seen that attacking important stations causes $R_{\text {path }}$ to decrease much more quickly than attacking stations randomly. In addition, $R_{\text {path }}$ decreases at a slower rate during non-peak hours than during peak hours when the same number of important stations are attacked using the same attack mode. Analyzing the influence of different attack modes on $R_{\text {path }}$ shows that $R_{\text {path }}$ is more sensitive to attacks on important stations as identified by $\mathrm{CC}, \mathrm{BC}$ and RAPV indicators. Attacking three important stations that are identified by BC, DC and RAPV indicators causes $R_{\text {path }}$ to drop below 0.3 during peak hours and to drop to nearly 0.6 during non-peak hours. Thus the BC, DC and RAPV indicators can effectively identify the important stations that have a large influence on $R_{\text {path }}$.

\subsubsection{Relative Travel Efficiency of Wuhan's subway network}

The shortest travel time between stations will increase when stations experience failure. Therefore, the connectivity reliability of Wuhan's subway network can be reflected by relative travel efficiency $R_{\text {eff }}$. Figure 3 shows relative travel efficiency $R_{\text {eff }}$ simulation outputs under five attack modes during peak and non-peak hours. Compared with Fig. 2, it shows that attacking the same number of important stations using the same attack mode causes $R_{\text {path }}$ to decrease more rapidly than $R_{\text {eff }}$. Therefore, station failures have a greater impact on passengers' tolerable travel paths than on passenger travel efficiency. It is found that 
Table 2 Transfer time at transfer stations during peak and non-peak hours

\begin{tabular}{|c|c|c|c|c|c|c|}
\hline \multirow[t]{2}{*}{ Stations } & \multirow[t]{2}{*}{ Transfer direction } & \multicolumn{2}{|l|}{$t^{\operatorname{trans}}(\min )$} & \multirow[t]{2}{*}{ Transfer direction } & \multicolumn{2}{|l|}{$t^{\text {trans }}(\min )$} \\
\hline & & Peak hours & Non-peak hours & & Peak hours & Non-peak hours \\
\hline 14 & Line $1-3$ & 2.92 & 2.07 & Line $3-1$ & 2.58 & 1.83 \\
\hline 20 & Line $1-2$ & 2.50 & 1.83 & Line $2-1$ & 2.00 & 1.62 \\
\hline 21 & Line $1-5$ & 2.67 & 2.00 & Line $5-1$ & 2.67 & 1.76 \\
\hline 23 & Line $1-6$ & 2.58 & 2.06 & Line $6-1$ & 2.67 & 2.19 \\
\hline 38 & Line $2-6$ & 2.00 & 1.50 & Line 6-2 & 1.67 & 1.25 \\
\hline 38 & Line $2-3$ & 2.50 & 1.90 & Line $3-2$ & 2.17 & 1.65 \\
\hline 41 & Line $2-5$ & 1.67 & 1.32 & Line $5-2$ & 2.08 & 1.68 \\
\hline 44 & Line $2-3$ & 2.58 & 2.06 & Line $3-2$ & 2.42 & 1.79 \\
\hline 48 & Line $2-5$ & 1.42 & 1.07 & Line $5-2$ & 1.75 & 1.31 \\
\hline 52 & Line $2-4$ & 1.83 & 1.32 & Line $4-2$ & 2.67 & 1.95 \\
\hline 53 & Line $2-4$ & 1.42 & 1.01 & Line 4-2 & 1.83 & 1.30 \\
\hline 61 & Line $3-5$ & 1.50 & 1.25 & Line $5-3$ & 2.33 & 1.70 \\
\hline 68 & Line $3-4$ & 2.75 & 2.31 & Line $4-3$ & 2.50 & 2.10 \\
\hline 73 & Line $3-5$ & 2.50 & 2.15 & Line $5-3$ & 1.67 & 1.27 \\
\hline 75 & Line $3-6$ & 1.92 & 1.29 & Line $6-3$ & 1.58 & 1.06 \\
\hline 79 & Line $3-7$ & 3.00 & 2.34 & Line $7-3$ & 2.17 & 1.71 \\
\hline 89 & Line $4-5$ & 1.42 & 1.09 & Line $5-4$ & 2.17 & 1.67 \\
\hline 98 & Line $4-6$ & 2.00 & 1.50 & Line $6-4$ & 2.25 & 1.69 \\
\hline
\end{tabular}

Table 3 Travel time for various links on line 1 during peak hours

\begin{tabular}{lllllll}
\hline Links (station-station) & \multicolumn{2}{l}{ Travel time (min) } & & Links (station-station) & \multicolumn{2}{c}{ Travel time (min) } \\
\cline { 2 - 3 } & Ds & Us & & Ds & Us \\
\hline $1-2$ & 1.90 & 1.71 & $6-7$ & $7-8$ & 1.62 & 1.23 \\
$2-3$ & 3.38 & 3.03 & & -8 & 1.38 & 1.62 \\
$3-4$ & 1.78 & 1.63 & & $8-9$ & 2.46 & 2.53 \\
$4-5$ & 2.83 & 2.79 & & $9-10$ & 2.60 & 3.06 \\
$5-6$ & 2.84 & 3.05 & & $10-11$ & 1.55 & 1.44 \\
\hline
\end{tabular}

Table 4 The 10 most important stations identified by important station identification indicators

\begin{tabular}{llllllll}
\hline Station number & DC & Station number & BC & Station number & CC & Station number & RAPV \\
\hline 38 & 5 & 75 & 0.026 & 21 & 11.05 & 21 & 0.030 \\
14 & 4 & 73 & 0.025 & 73 & 11.12 & 48 & 0.026 \\
20 & 4 & 21 & 0.023 & 20 & 11.16 & 20 & 0.024 \\
21 & 4 & 14 & 0.021 & 118 & 11.29 & 23 & 0.022 \\
23 & 4 & 48 & 0.020 & 22 & 11.32 & 73 & 0.022 \\
41 & 4 & 98 & 0.019 & 74 & 11.35 & 75 & 0.022 \\
44 & 4 & 68 & 0.019 & 48 & 11.43 & 14 & 0.020 \\
48 & 4 & 23 & 0.017 & 72 & 11.50 & 44 & 0.016 \\
68 & 4 & 89 & 0.016 & 23 & 11.51 & 52 & 0.016 \\
73 & 4 & 76 & 0.015 & 75 & 11.54 & 89 & 0.016 \\
\hline DC = degree centrality, BC = betweenness centrality, CC = closeness centrality, RAPV = ratio of affected \\
passenger volume
\end{tabular}




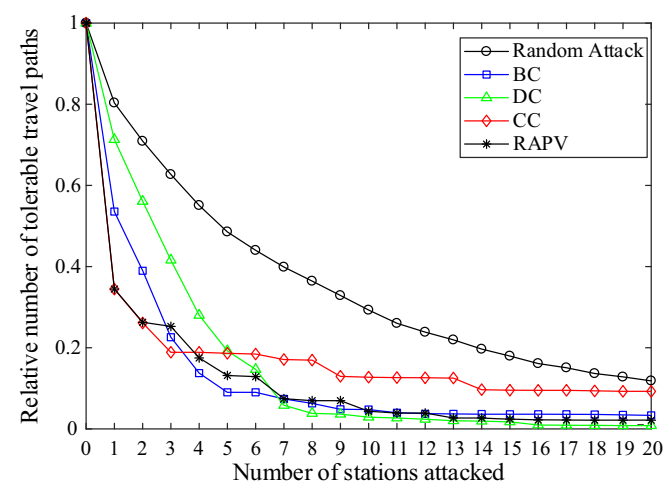

(a)

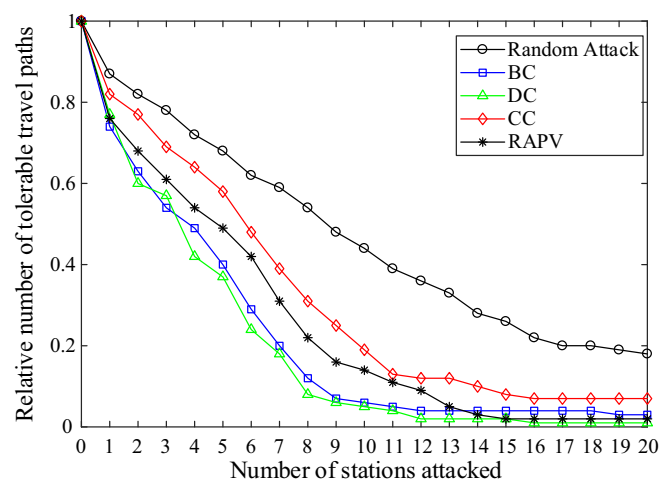

(b)

Fig. 2 Simulation output showing relative number of tolerable travel paths $R_{\text {path }}$ under five attack modes a during peak hours and $\mathbf{b}$ during nonpeak hours

attacking important stations deliberately causes $R_{\text {eff }}$ to decrease much more quickly than attacking stations randomly. The rate at which $R_{\text {eff }}$ decreases during non-peak hours is less than that during peak hours when the same number of important stations are attacked using the same attack mode. This means that passenger travel efficiency during non-peak hours will remain higher than during peak hours when station failures are the same. In addition, $R_{\text {eff }}$ is sensitive to attacks on important stations as identified by BC, DC and RAPV indicators. Therefore, these three indicators effectively identify important stations that have a significant influence on travel efficiency.

\subsubsection{Rate of Passenger Travel Realization on Wuhan's subway network}

Some passengers' tolerable travel paths will be completely interrupted when stations in a URT network are destroyed. In such a case, the travel time of other connected paths will exceed passengers' tolerable travel time, and some passengers will choose alternative transportation. Therefore, the connectivity reliability of Wuhan's subway network

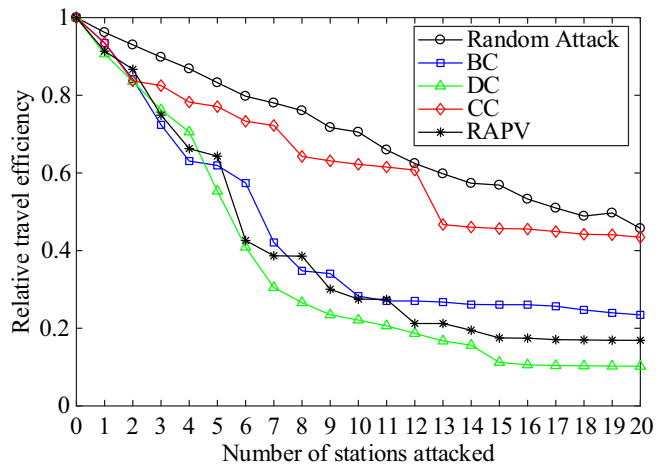

(a) can be reflected by the rate of passenger travel realization $R_{\text {rate }}$. Figure 4 shows the rate of passenger travel realization $R_{\text {rate }}$ from simulation output under five attack modes during peak and non-peak hours. Compared with Fig. 2, it shows that the passenger travel realization rate decreases more slowly than the relative number of tolerable travel paths when stations are destroyed, which proves that attacks on stations have a greater impact on passengers' tolerable travel paths than on passenger travel realization. In addition, the rate of passenger travel realization on the Wuhan subway decreases more slowly during non-peak hours than during peak hours when the same number of important stations are attacked with the same attack mode. Therefore, station failures have less impact on passenger travel realization during non-peak hours. A comparison of the impact of different attack modes on $R_{\text {rate }}$ shows that attacking three important stations as identified by BC, DC and RAPV indicators causes $R_{\text {rate }}$ to decrease to less than 0.8 , which means that more than $20 \%$ of passengers cannot travel to their destination within tolerable travel time. Among the five attack modes, stations identified by the RAPV indicator show the fastest decrease in $R_{\text {rate }}$ during an attack,

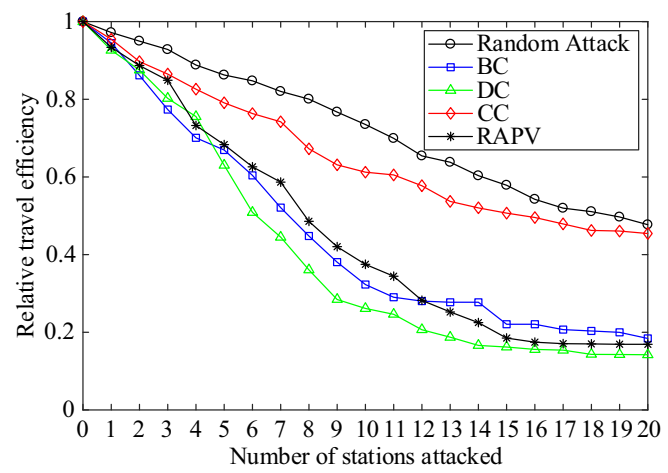

(b)

Fig. 3 Simulation output showing relative travel efficiency of the Wuhan subway under five attack modes a during peak hours and b during nonpeak hours 


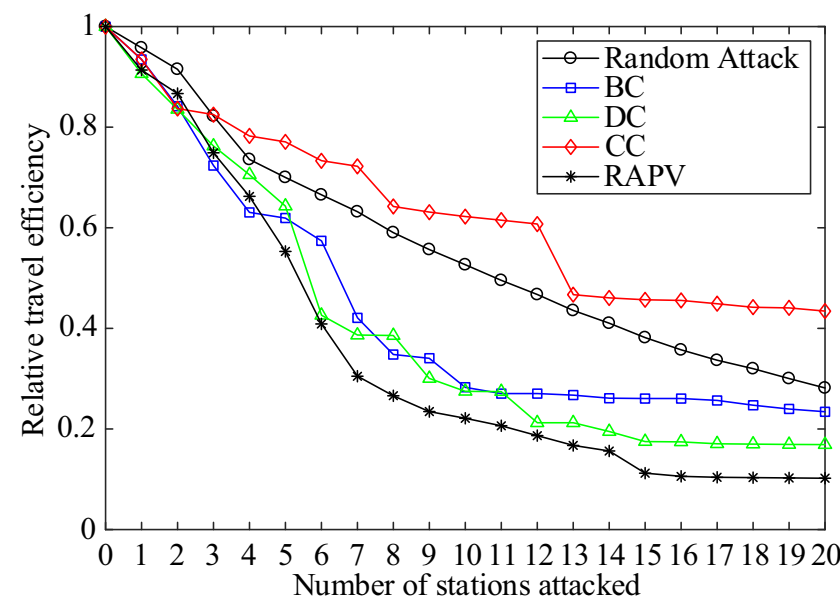

(a)

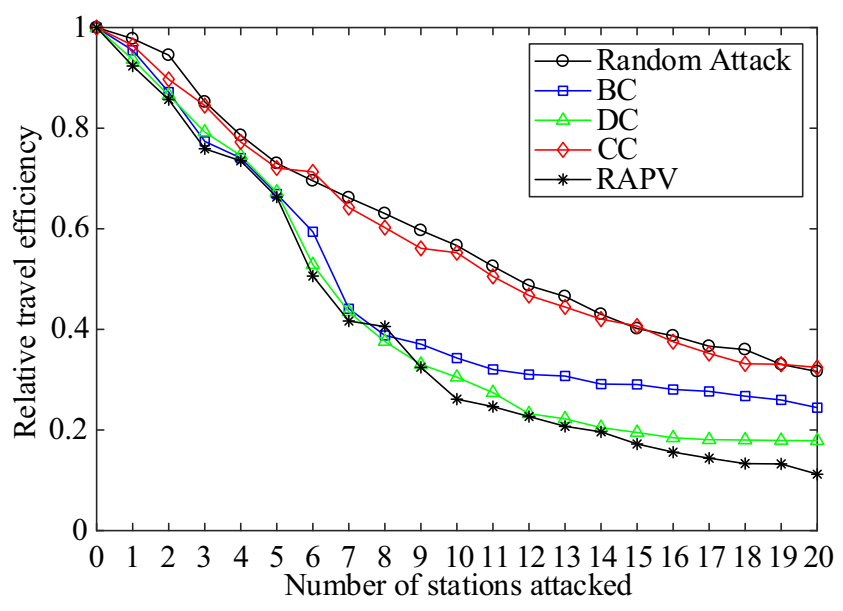

(b)

Fig. 4 Passenger travel realization simulation output under five attack modes a during peak hours and $\mathbf{b}$ during non-peak hours

which demonstrates that the RAPV indicator is effective in identifying important stations with a strong influence on $R_{\text {rate }}$.

\subsubsection{Sensitivity Analysis of the Passenger Tolerability Coefficient}

The average number of tolerable travel paths and the rate of passenger travel realization on Wuhan's subway network are closely related to the passenger tolerability coefficient: the higher the tolerability coefficient value, the higher the average number of tolerable travel paths and the higher the rate of passenger travel realization. Therefore, an analysis was performed to determine the sensitivity of the tolerability coefficient (from 1 to 1.6) with respect to the average number of tolerable travel paths and the rate of passenger travel realization. The passenger tolerability coefficient is greater than or equal to 1 . All passengers travel on a URT using the fastest travel paths when the passenger tolerability coefficient is equal to 1 . In this case, if the fastest travel path is interrupted between a pair of stations, then passengers cannot travel on a URT network between these stations.

The sensitivity of the passenger tolerability coefficient to the average number of tolerable travel paths on Wuhan's subway network during peak hours is shown in Fig. 5. Figure 5a-d shows the sensitivity when different numbers of stations are attacked using different attack modes. Conclusions are obtained by analyzing the sensitivity of the tolerability coefficient to the average number of tolerable travel paths during peak and non-peak hours, as follows: The average number of tolerable travel paths during peak hours is sensitive to changes in the passenger tolerability coefficient when stations are attacked. The higher the value of the passenger tolerability coefficient, the higher the average number of tolerable travel paths and the higher the connectivity reliability of Wuhan's subway network. As the number of stations attacked increases, the sensitivity of the passenger tolerability coefficient to the average number of tolerable travel paths decreases rapidly. The average number of tolerable travel paths on Wuhan's subway network is most sensitive to changes in the passenger tolerability coefficient when attacks occur on important stations as identified by the DC and RAPV indicators.

The sensitivity of the passenger tolerability coefficient to the rate of passenger travel realization on Wuhan's subway network during peak hours is shown in Fig. 6. Figure 6a-d shows the sensitivity when different numbers of stations are attacked using different attack modes. Conclusions are obtained by analyzing the sensitivity of the tolerability coefficient to the passenger travel realization rate during peak and non-peak hours, as follows: The passenger travel realization rate during peak hours is sensitive to the passenger tolerability coefficient. The travel realization rate is most sensitive to the passenger tolerability coefficient when attacks occur on important stations identified as by the BC, DC and RAPV indicators. Therefore, the higher the value of the passenger tolerability coefficient, the higher the passenger travel realization rate and the higher the connectivity reliability of Wuhan's subway network.

Analysis of the effects of the passenger tolerability coefficient on the average number of tolerable travel paths and the rate of passenger travel realization on Wuhan's subway network shows that the passenger tolerability coefficient has a large impact on the connectivity reliability of the network. Therefore, a good travel experience (providing a clean vehicle, good travel instructions, good information service, discount policy for tickets during peak hours) should be provided to increase the passenger 


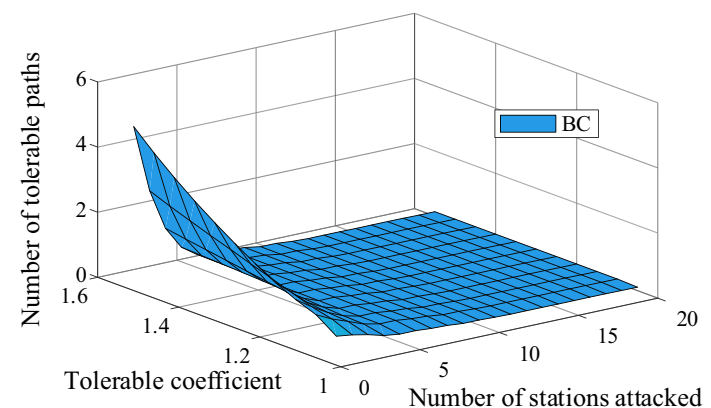

(a)

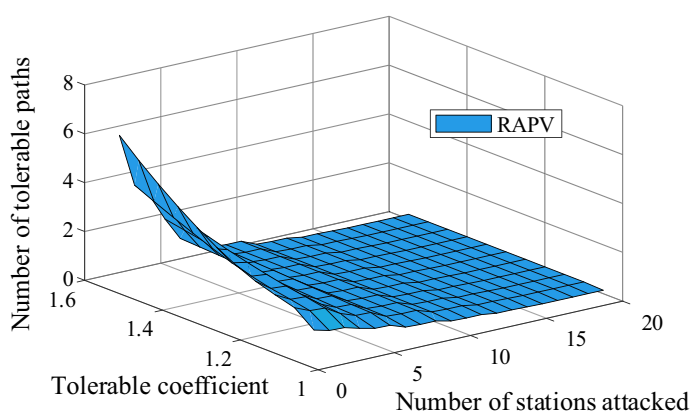

(c)

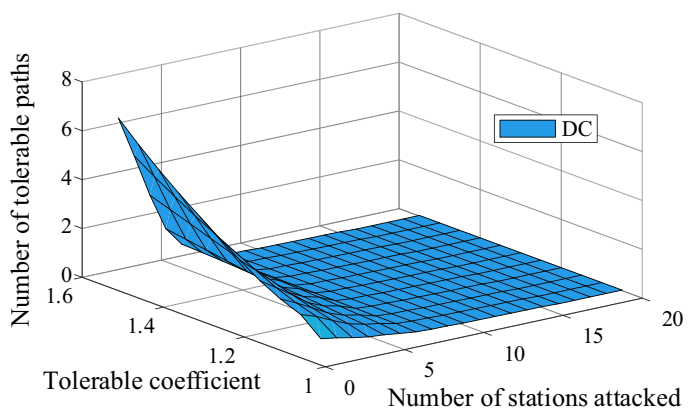

(b)

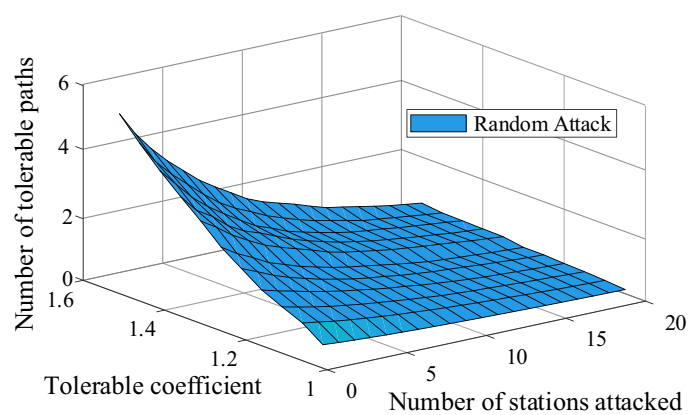

(d)

Fig. 5 Sensitivity of passenger tolerability coefficient to the average number of tolerable travel paths on Wuhan's subway network during peak hours

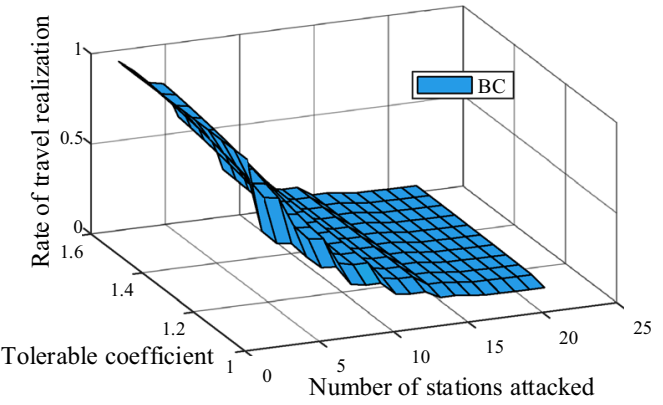

(a)

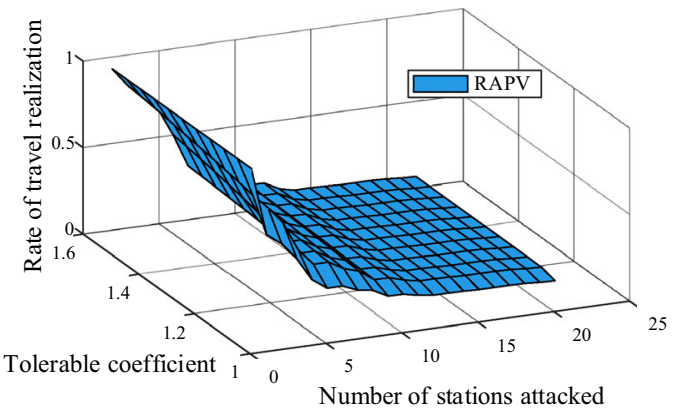

(c)

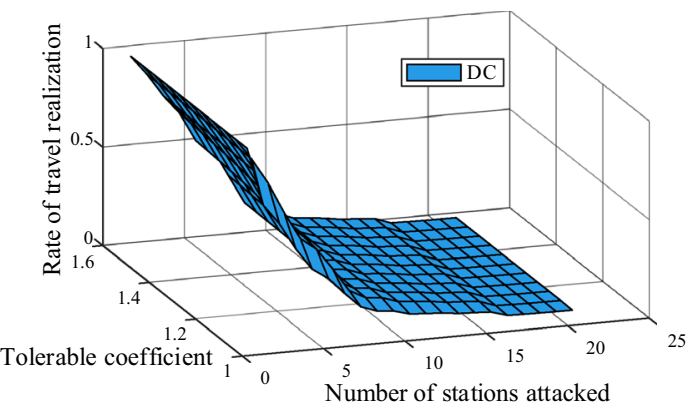

(b)

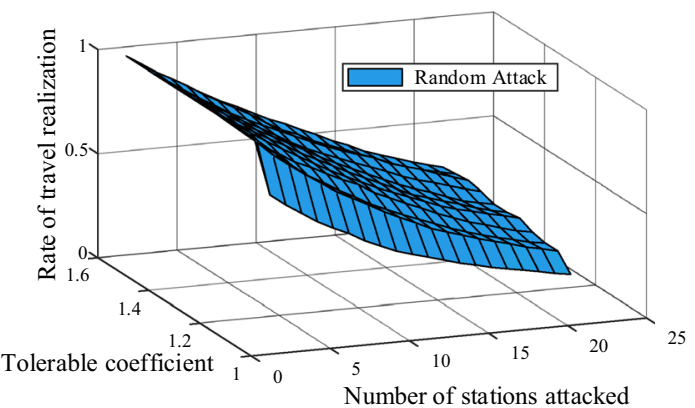

(d)

Fig. 6 Sensitivity analysis of the effect of the passenger tolerability coefficient on the rate of tolerable travel paths on Wuhan's subway network during peak hours 
Fig. 7 The 10 most important stations identified by DC, BC and RAPV indicators

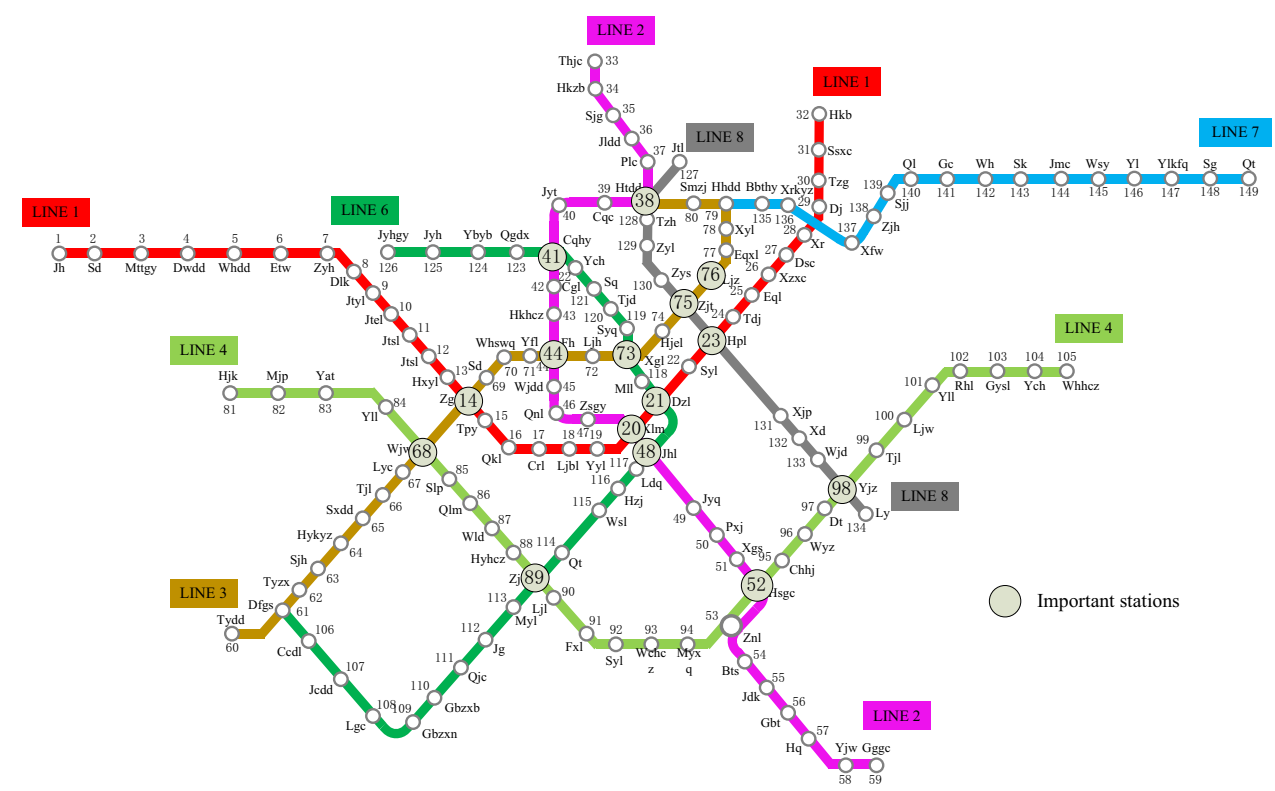

tolerability coefficient and thus effectively enhance the connectivity reliability of Wuhan's subway network.

\subsubsection{Important Stations for Connectivity Reliability of Wuhan's subway network}

From Figs. 2, 3 and 4, it is found that the DC, BC and RAPV indicators are able to effectively identify the important stations that have a large influence on the relative number of tolerable travel paths. The DC and RAPV indicators identify important stations that have a significant influence on relative travel efficiency and the rate of travel realization, respectively. From a general perspective, the DC, BC and RAPV indicators are able to identify important stations that have a large impact on different connectivity reliability evaluation indicators. In addition, Figs. 2, 3 and 4 show that the connectivity reliability evaluation indicators decrease rapidly when the number of stations attacked ranks between 1 and 10. This indicates that the 10 most important stations as identified by the DC, BC and RAPV indicators should be protected to maintain the connectivity reliability of Wuhan's subway network.

The 10 most important stations as identified by DC, BC and RAPV indicators are shown in Fig. 7. It can be seen that many of the important stations are located at the center of the city (stations 44, 20, 21, 23, 73, 75 and 76). Many passengers' travel paths contain these stations. The betweenness of these stations is high. Therefore, if these stations are attacked, the volume of affected passengers increases quickly, and the travel realization decreases rapidly. In addition, the important stations all contain bridge nodes (stations 14, 68, 89, 48, 52, 23 and 98). These stations play a significant role in connecting subgraphs of
Wuhan's subway network. If these stations are attacked, the network is divided into several subgraphs, and the connectivity of the network is severely disrupted. The above analysis reveals that stations that are important to the connectivity reliability of the Wuhan subway have certain features: the stations are located in the central region of the city, and the stations are important for connecting the subgraphs of the network.

\section{Conclusions}

In this work, the connectivity reliability of a URT network is measured based on passengers' tolerable travel paths, passenger travel efficiency and passenger travel realization. DC, BC, CC and RAPV indicators are used to identify the important stations in Wuhan's subway network. Finally, the connectivity reliability of Wuhan's subway network is analyzed by simulation of attacks on stations. Some findings and conclusions are summarized below:

- The connectivity reliability of Wuhan's subway network is more sensitive to attacking important stations than attacking stations randomly. The DC, BC and RAPV indicators can effectively identify important stations that have a huge influence on the connectivity reliability of the network.

- The new indicator RAPV can effectively identify the important stations with regard to the connectivity reliability of Wuhan's subway network. In particular, it can effectively identify the stations which have a significant influence on passenger travel realization on the network. 
- The connectivity of Wuhan's subway network is more reliable during non-peak hours than during peak hours. The connectivity reliability of the network is sensitive to the changes in the passenger tolerability coefficient when stations are attacked: the higher the value of the passenger tolerability coefficient, the higher the connectivity reliability of the network.

- The stations that are important to the connectivity reliability of the Wuhan subway have certain features: they are located at the center of the city, and they are important for connecting the subgraphs of the network.

Analyzing the connectivity reliability of a URT network and identifying important stations are of great importance to the normal operations of the network. Connectivity reliability can be improved by analyzing the influence of important stations on the network and carrying out reasonable protective measures. Although the connectivity reliability of one URT network is analyzed here, the same indicators and method can be used to analyze other URT networks. Future studies will combine additional aspects. For example, the probability of station failures can be determined by historical data, and the reliability of a URT network can be evaluated from the travel quality of passengers. In the future, the reliability of URT operations can be further improved by combining the connectivity reliability analysis with the optimization of train timetabling (Zhang et al. [29, 30]) and rolling stock scheduling (Zhong et al. [31]) of URT networks.
Acknowledgements The authors thank Wuhan Metro Co., Ltd. for providing relevant data. We also acknowledge the support of the National Key R \& D Program of China (2017YFB1200700) and the National Natural Science Foundation of China (no. U1834209).

Author Contributions Liu and Peng conceived and designed the study. Liu, Peng, Chen and Yin performed the simulation. Liu and Yin wrote the paper. Liu, Peng, Chen and Yin reviewed and edited the manuscript. All authors read and approved the manuscript.

Data Availability Some or all data, models or code generated or used during the study are available from the corresponding author by request.

\section{Compliance with Ethical Standards}

Conflict of interest The authors declare that they have no competing financial interests.

Open Access This article is distributed under the terms of the Creative Commons Attribution 4.0 International License (http://crea tivecommons.org/licenses/by/4.0/), which permits unrestricted use, distribution, and reproduction in any medium, provided you give appropriate credit to the original author(s) and the source, provide a link to the Creative Commons license, and indicate if changes were made.

\section{Appendix}

The travel time for all links in Wuhan's subway network during peak hours is shown as Table 5.

Table 5 Travel time for all links in Wuhan's subway network during peak hours

\begin{tabular}{|c|c|c|c|c|c|c|c|c|}
\hline \multirow[t]{2}{*}{ Links } & \multicolumn{2}{|c|}{ Travel time (min) } & \multirow[t]{2}{*}{ Links } & \multicolumn{2}{|c|}{ Travel time (min) } & \multirow[t]{2}{*}{ Links } & \multicolumn{2}{|c|}{ Travel time (min) } \\
\hline & Ds & Us & & Ds & Us & & Ds & Us \\
\hline $1-2$ & 1.90 & 1.71 & $53-54$ & 2.43 & 2.91 & $102-103$ & 2.8 & 3.42 \\
\hline $2-3$ & 3.38 & 3.03 & $54-55$ & 2.12 & 1.8 & $103-104$ & 2.01 & 2.2 \\
\hline $3-4$ & 1.78 & 1.63 & $55-56$ & 1.63 & 1.3 & $104-105$ & 1.39 & 1.13 \\
\hline $4-5$ & 2.83 & 2.79 & $56-57$ & 2.77 & 2.5 & $61-106$ & 2.22 & 2.47 \\
\hline $5-6$ & 2.84 & 3.05 & $57-58$ & 2.47 & 2.42 & $106-107$ & 3.91 & 3.15 \\
\hline $6-7$ & 1.62 & 1.23 & $58-59$ & 2.26 & 1.81 & $107-108$ & 4.81 & 3.89 \\
\hline $7-8$ & 1.38 & 1.62 & $60-61$ & 2.48 & 3.1 & $108-109$ & 3.13 & 3.35 \\
\hline $8-9$ & 2.46 & 2.53 & $61-62$ & 1.44 & 1.32 & $109-110$ & 1.3 & 1.19 \\
\hline $9-10$ & 2.60 & 3.06 & $62-63$ & 2.49 & 2.24 & $110-111$ & 2.61 & 2.82 \\
\hline $10-11$ & 1.55 & 1.44 & $63-64$ & 2.68 & 2.09 & $111-112$ & 1.63 & 1.84 \\
\hline $11-12$ & 1.36 & 1.33 & $64-65$ & 1.59 & 1.43 & 89-113 & 2.2 & 2.29 \\
\hline $12-13$ & 1.41 & 1.09 & $65-66$ & 2.33 & 1.8 & $112-113$ & 2.53 & 2.84 \\
\hline $13-14$ & 1.58 & 1.32 & $66-67$ & 1.55 & 1.56 & 89-114 & 1.6 & 1.39 \\
\hline $14-15$ & 2.71 & 2.93 & $14-68$ & 4.34 & 4.91 & $114-115$ & 3.18 & 3.56 \\
\hline $15-16$ & 1.79 & 1.64 & $67-68$ & 1.9 & 2.02 & $115-116$ & 1.31 & 1.61 \\
\hline $16-17$ & 1.96 & 2.35 & $14-69$ & 1.68 & 1.34 & $48-117$ & 1.46 & 1.73 \\
\hline $17-18$ & 1.51 & 1.22 & $69-70$ & 3.13 & 2.47 & $116-117$ & 1.67 & 1.33 \\
\hline 18-19 & 1.52 & 1.89 & $44-71$ & 1.52 & 1.73 & 21-118 & 1.38 & 1.29 \\
\hline
\end{tabular}


Table 5 continued

\begin{tabular}{|c|c|c|c|c|c|c|c|c|}
\hline \multirow[t]{2}{*}{ Links } & \multicolumn{2}{|c|}{ Travel time (min) } & \multirow[t]{2}{*}{ Links } & \multicolumn{2}{|c|}{ Travel time (min) } & \multirow[t]{2}{*}{ Links } & \multicolumn{2}{|c|}{ Travel time (min) } \\
\hline & Ds & Us & & Ds & Us & & Ds & Us \\
\hline 19-20 & 1.69 & 1.72 & $70-71$ & 2.1 & 2.53 & $73-118$ & 1.48 & 1.38 \\
\hline $20-21$ & 1.86 & 2.05 & $44-72$ & 2.87 & 2.92 & $73-119$ & 1.76 & 1.92 \\
\hline $21-22$ & 1.73 & 2.16 & $72-73$ & 1.6 & 1.29 & $119-120$ & 1.63 & 1.71 \\
\hline $22-23$ & 1.98 & 1.77 & $73-74$ & 1.6 & 1.86 & $120-121$ & 2.53 & 2.9 \\
\hline $23-24$ & 2.06 & 1.97 & $23-75$ & 2.43 & 2.24 & $41-122$ & 3.62 & 3.38 \\
\hline $24-25$ & 1.57 & 1.54 & $74-75$ & 1.54 & 1.38 & $121-122$ & 1.45 & 1.23 \\
\hline $25-26$ & 1.36 & 1.54 & $75-76$ & 1.51 & 1.7 & $41-123$ & 1.65 & 1.31 \\
\hline $26-27$ & 2.61 & 3.03 & $76-77$ & 1.3 & 0.98 & $123-124$ & 2.06 & 2.34 \\
\hline $27-28$ & 2.46 & 1.97 & $77-78$ & 2.15 & 1.66 & $124-125$ & 3 & 2.56 \\
\hline $28-29$ & 2.00 & 1.68 & $78-79$ & 2.02 & 2.19 & $125-126$ & 4.1 & 3.87 \\
\hline $29-30$ & 1.76 & 1.63 & $38-80$ & 4.15 & 4.37 & $38-127$ & 1.47 & 1.5 \\
\hline $30-31$ & 1.92 & 1.49 & $79-80$ & 2.86 & 2.9 & $38-128$ & 3.24 & 2.8 \\
\hline $31-32$ & 5.85 & 5.91 & $81-82$ & 4.73 & 5.28 & $128-129$ & 2.83 & 3.03 \\
\hline $33-34$ & 10.29 & 9.45 & $82-83$ & 2.54 & 2.8 & $75-130$ & 2.39 & 2.37 \\
\hline $34-35$ & 2.44 & 2.05 & $68-84$ & 1.54 & 1.76 & 129-130 & 2.7 & 2.23 \\
\hline $35-36$ & 3.50 & 2.99 & $83-84$ & 2.13 & 1.9 & 23-131 & 5.99 & 6.83 \\
\hline $36-37$ & 2.82 & 3.39 & $68-85$ & 1.63 & 1.79 & $131-132$ & 1.7 & 1.36 \\
\hline $37-38$ & 6.76 & 7.36 & $85-86$ & 2.06 & 2.12 & $98-133$ & 2.26 & 2.03 \\
\hline $38-39$ & 4.95 & 4.87 & $86-87$ & 1.93 & 1.83 & $132-133$ & 1.28 & 1.11 \\
\hline $39-40$ & 3.45 & 4.16 & $87-88$ & 1.73 & 1.35 & 98-134 & 1.5 & 1.52 \\
\hline $40-41$ & 1.87 & 1.50 & $88-89$ & 2.7 & 3.08 & 79-135 & 1.69 & 1.34 \\
\hline $41-42$ & 2.96 & 3.32 & $89-90$ & 1.43 & 1.31 & $135-136$ & 3.02 & 2.87 \\
\hline $42-43$ & 2.41 & 2.70 & $90-91$ & 5.62 & 5.92 & $136-137$ & 3.55 & 2.85 \\
\hline $43-44$ & 2.08 & 2.15 & $91-92$ & 1.23 & 1.38 & $137-138$ & 1.97 & 1.59 \\
\hline $44-45$ & 2.42 & 2.04 & $92-93$ & 1.54 & 1.23 & 138-139 & 2.04 & 2.33 \\
\hline $45-46$ & 1.72 & 1.80 & 53-94 & 1.88 & 1.53 & $139-140$ & 4.04 & 3.62 \\
\hline $20-47$ & 2.65 & 2.38 & $93-94$ & 1.54 & 1.58 & $140-141$ & 2.13 & 2.24 \\
\hline $46-47$ & 1.62 & 1.33 & $52-95$ & 1.85 & 1.83 & $141-142$ & 4.15 & 5.11 \\
\hline $20-48$ & 1.54 & 1.32 & $95-96$ & 2.1 & 2.51 & $142-143$ & 6.35 & 6.13 \\
\hline $21-48$ & 2.66 & 3.19 & $96-97$ & 1.8 & 2.07 & $143-144$ & 1.88 & 2.06 \\
\hline $48-49$ & 5.64 & 4.43 & 97-98 & 1.59 & 1.78 & $144-145$ & 4.82 & 5.44 \\
\hline $49-50$ & 2.71 & 2.36 & 98-99 & 1.77 & 1.37 & $145-146$ & 3.31 & 3.2 \\
\hline $50-51$ & 1.59 & 1.24 & 99-100 & 2.01 & 1.58 & $146-147$ & 4.69 & 5.06 \\
\hline $51-52$ & 2.00 & 1.94 & $100-101$ & 1.78 & 1.41 & $147-148$ & 3.36 & 2.7 \\
\hline $52-53$ & 3.31 & 2.51 & $101-102$ & 2.29 & 2.63 & $148-149$ & 4.15 & 5.04 \\
\hline
\end{tabular}

Ds: downstream, Us: upstream

\section{References}

1. Latora V, Marchiori M (2002) Is the Boston subway a smallworld network? Physica A 314(1-4):109-113

2. Derrible S, Kennedy C (2010) The complexity and robustness of metro networks. Physica A 389(17):3678-3691

3. Seaton KA, Hackett LM (2004) Stations, trains and small-world networks. Physica A 339(3-4):635-644
4. Hosseini S, Wadbro E (2016) Connectivity reliability in uncertain networks with stability analysis. Expert Syst Appl 57:337-344

5. Zhang YS, Yao EJ (2015) Splitting travel time based on AFC data: estimating walking, waiting, transfer, and in-vehicle travel time in metro system. Discrete Dyn Nat Soc 2015:1-11

6. Du MQ, Jiang XW, Cheng L et al (2017) Robust evaluation for transportation network capacity under demand uncertainty. J Adv Transp 2017:1-11

7. Mine H, Kawai H (1982) Mathematics for reliability analysis. Asakura-shorten, Tokyo

8. Bell MGH, Iida Y (1997) Transportation network analysis. Wiley, Chichester 
9. Wakabayashi H, Iida Y (1992) Upper and lower bounds of terminal reliability in road networks: an efficient method with Boolean algebra. J Nat Disaster Sci 14:29-44

10. Jiang CZ, Xu F, Yuan JX (2013) Characteristics and reliability analysis of the complex network in Guangzhou rail transit. Intell Autom Soft Comput 19(2):217-225

11. Liu ZQ, Song R (2010) Reliability analysis of Guangzhou rail transit with complex network theory. J Transp Syst Eng Inf Technol 10(5):194-200

12. Wang ZQ, Xu RH (2009) Reliability simulation analysis of urban rail transit networks based on complex network. J Syst Simul 21(20):6670-6674

13. Zhang JH, Hong L, Wang SL, et al (2011) Reliability assessments of Chinese highspeed railway network. In: IEEE international conference on intelligent rail transportation, Beijing, China

14. Zhang X, Miller-Hooks E, Denny K (2015) Assessing the role of network topology in transportation network resilience. J Transp Geogr 46:35-45

15. Mattsson LG, Jenelius E (2015) Vulnerability and resilience of transport systems - A discussion of recent research. Transp Res Part A 81:16-34

16. Zhang X, Jia L, Dong H, et al (2009) Analysis and evaluation of connectivity reliability for dynamic transportation network. In: 2009 Fifth international joint conference on INC, IMS and IDC. Seoul, South Korea

17. Liu J, Lu H, Ma H et al (2017) Network vulnerability analysis of rail transit plans in Beijng-Tianjin-Hebei region considering connectivity reliability. Sustainability $9: 1479$

18. Guidotti R, Gardoni P, Chen Y (2017) Network reliability analysis with link and nodal weights and auxiliary nodes. Struct Saf 65:12-26

19. Li M, Jia L, Wang Y (2015). Research and implementation on connectivity reliability calculation algorithm of Urban Rail Transit network operation. In: Proceeding of the 11th world congress on intelligent control and automation, Shenyang, China

20. Reggiani A, Nijkamp P, Lanzi D (2015) Transport resilience and vulnerability: the role of connectivity. Transp Res Part A 81:4-15
21. Liu J, Xiong Q, Shi W et al (2016) Evaluating the importance of nodes in complex networks. Phys A 452:209-219

22. Rodriguez-Nunez E, Garcia-Palomares JC (2014) Measuring the vulnerability of public transport networks. J Transp Geogr 35:50-63

23. Hu P, Fan W, Mei S (2015) Identifying node importance in complex networks. Phys A 429:169-176

24. Liu J, Zhou X (2016) Capacitated transit service network design with boundedly rational agents. Transp Res Part B Methodol $3: 225-250$

25. Shang P, Li R, Guo J et al (2019) Integrating Lagrangian and Eulerian observations for passenger flow state estimation in an urban rail transit network: a space-time-state hyper networkbased assignment approach. Transp Res Part B Methodol 121:35-167

26. Zhu W, Hu H, Xu R et al (2013) Modified stochastic user-equilibrium assignment algorithm for urban rail transit under network operation. J Cent South Univ 20(10):2897-2904

27. Han B, Zhou W, Li D et al (2015) Dynamic schedule-based assignment model for urban rail transit network with capacity constraints. Sci World J 2015:1-12

28. Wardman M, Whelan G (2011) Twenty years of rail crowding valuation studies: evidence from lessons from British experience. Transp Rev 31(3):379-398

29. Zhang Y, D’Ariano A, He B, Peng Q (2019) Microscopic optimization model and algorithm for integrating train timetabling and track maintenance task scheduling. Transp Res Part B Methodol 127:237-278

30. Zhang Y, Peng Q, Yao Y, Zhang X, Zhou X (2019) Solving cyclic train timetabling problem through model reformulation: extended time-space network construct and alternating direction method of multipliers methods. Transp Res Part B Methodol 128:344-379

31. Zhong Q, Lusby RM, Larsen J, Zhang Y, Peng Q (2019) Rolling stock scheduling with maintenance requirements at the Chinese high-speed railway. Transp Res Part B Methodol 126:24-44 